\title{
A test of Darwin's naturalization conundrum in birds reveals enhanced invasion success in presence of close relatives
}

\author{
Daniel Sol ${ }^{1}$, Joan Garcia-Porta ${ }^{2}$, César González-Lagos ${ }^{3}$, Alex Pigot ${ }^{4}$, Joseph Tobias ${ }^{5}$, and \\ Christopher Trisos ${ }^{6}$ \\ ${ }^{1} \mathrm{CREAF}$ \\ ${ }^{2}$ Universitat Autonoma de Barcelona Centre de Recerca Ecologica i Aplicacions Forestals \\ ${ }^{3}$ Universidad Bernardo O'Higgins \\ ${ }^{4}$ University College London Division of Biosciences \\ ${ }^{5}$ Imperial College London \\ ${ }^{6}$ University of Maryland at College Park
}

February 22, 2021

\begin{abstract}
Despite biological invasions are one of the main environmental problems of the twenty-first century, there is still no theoretical or empirical agreement on whether a high phylogenetic relatedness between exotic and native species positively or negatively affect invasion success. To resolve this conundrum, it has been proposed that the effect might be scale-dependent, being negative at smaller spatial scales and positive at larger scales. Here we show that this scale-dependent pattern may be a sampling artefact associated with species-area effects and a non-random pattern of species introductions. We support this conclusion with simulations and empirical data on invaded and non-invaded avian communities in regions from five continents. We further show that at smaller-scales - where these artifacts are negligible- invasion success generally increases with the presence of closely-related species, but that predictive accuracy largely depends on considering the influence of human-related disturbances in facilitating invasions.
\end{abstract}

A test of Darwin's naturalization conundrum in birds reveals enhanced invasion success in presence of close relatives

Daniel Sol ${ }^{1,2^{*}}$, Joan Garcia-Porta ${ }^{2,3^{*}}$, Cesar González-Lagos ${ }^{4,5}$, Alex L. Pigot ${ }^{6}$, Joseph A. Tobias ${ }^{7}$ \& Christopher Trisos ${ }^{8,9}$

${ }^{1}$ CSIC, Spanish National Research Council, CREAF-UAB, Catalonia 08193, Spain.

${ }^{2}$ CREAF, Centre for Ecological Research and Applied Forestries, Catalonia 08193, Spain.

${ }^{3}$ Department of Biology, Washington University in St Louis, USA

${ }^{4}$ Centro de Investigación en Recursos Naturales y Sustentabilidad (CIRENYS),

Universidad Bernardo O'Higgins, Santiago, Chile.

${ }^{5}$ Center of Applied Ecology and Sustainability (CAPES).

${ }^{6}$ Centre for Biodiversity and Environment Research, Department of Genetics,

Evolution and Environment, University College London, London WC1E 6BT,

$U K$ 
${ }^{7}$ Department of Life Sciences, Imperial College London, Silwood Park, Buckhurst Road, Ascot, UK

${ }^{8}$ African Climate and Development Initiative (ACDI), University of Cape Town, South Africa.

${ }^{9}$ Centre for Statistics in Ecology, Environment and Conservation, University of Cape Town, Cape Town, South Africa.

* Shared first authorship

Number of words in the abstract (144)

Number of words in the manuscript as a whole, excluding abstract, references, and captions (4550)

Number of references in main text (51) Number of figures (5) and tables (0) in main text

Name and complete mailing address of the person to whom correspondence should be sent: Daniel Sol, CREAF-CSIC, Cerdanyola del Vallès, Catalonia E-08193, Spain. TEL: + 34 93-5814678. E-MAIL: d.sol@creaf.uab.cat

Author contributions: DS, JGC and CGL conceived and designed the study; DS, CGL, ALP, JAT and CT collected data; DS and JGP conducted the analyses; DS wrote the manuscript and all authors edited and approved it.

Data accessibility statement: We confirm that, should the manuscript be accepted, the data supporting the results will be archived Dryad, and the data DOI will be included at the end of the article.

Abstract. Despite biological invasions are one of the main environmental problems of the twenty-first century, there is still no theoretical or empirical agreement on whether a high phylogenetic relatedness between exotic and native species positively or negatively affect invasion success. To resolve this conundrum, it has been proposed that the effect might be scale-dependent, being negative at smaller spatial scales and positive at larger scales. Here we show that this scale-dependent pattern may be a sampling artefact associated with species-area effects and a non-random pattern of species introductions. We support this conclusion with simulations and empirical data on invaded and non-invaded avian communities in regions from five continents. We further show that at smaller-scales - where these artifacts are negligible- invasion success generally increases with the presence of closely-related species, but that predictive accuracy largely depends on considering the influence of human-related disturbances in facilitating invasions.

Keywords: Biotic resistance; environmental filtering; invasion potential; risk assessment; alien species

Growing concern over the environmental and socio-economic impact of biological invasions has fueled a surge of interest in identifying and preventing situations where the risk of a species becoming invasive is high (Hulme et al. 2008; Blackburn et al. 2009; Pyšek \& Richardson 2010; Pysek et al. 2010; Li et al. 2015). However, anticipating these situations has proved challenging, primarily because the risk of invasion depends not only on the features of the exotic species but also on the way they interact with the species from the recipient community (Shea \& Chesson 2002; Duncan et al.2003; Romanuk et al. 2009). A potential way to tackle this challenge was proposed by Darwin (Darwin 1859) who suggested that the degree of evolutionary relatedness between exotic and native species can inform the likelihood that a species establishes and proliferates (i.e. becomes invasive). If invasion success were related to evolutionary relatedness, this would not only allow a more accurate assessment of the risk of biological invasions but also assess the risk for species that are data-deficient regarding their ecology or success in previous introductions.

Although Darwin's suggestion has attracted increased research attention, there is still no empirical agreement on whether relatedness to native species positively or negatively influences invasion success (Cadotteet al. 2018). In fact, both possibilities are predicted by theory (Darwin 1859; Daehler 2001; Thuiller et al. 2010; Cadotteet al. 2018). Darwin's naturalization hypothesis suggests that introduced species should be less successful at invading communities in which their close relatives are present, because phylogenetically-related species often share similar niches and hence are more likely to compete for similar resources. Thus, the hypothesis predicts a negative association between relatedness to native species and invasion success. However, 
if successful invasion instead primarily depends on phylogenetically-conserved adaptations to find a suitable niche in the novel environment, then the presence of close-relatives may indicate that the invader already has the necessary adaptations (hereafter 'preadaptation hypothesis'). This predicts a positive association between relatedness to native species and invasion success.

Both the naturalization and preadaptation hypotheses are theoretically sound and have been demonstrated in small-scale experiments (Jianget al. 2010; Li et al. 2015), but extrapolating the results to the real world has proven difficult, with multiple studies reporting contradictory results (Cadotte et al. 2018). Discrepancies among studies may reflect methodological shortcomings (Thuiller et al. 2010; Li et al. 2015). These include inaccurate assessment of invasion success, insufficient phylogenetic resolution, inappropriate spatial scales of analysis and violation of the assumption of statistical independence when making inference (Lambdon \& Hulme 2006; Diez et al. 2008; Thuiller et al.2010; Sol et al. 2014b; Li et al. 2015). Thuiller et al. (2010), for example, suggested that large spatial scales and taxonomy-based relatedness metrics are unlikely to detect the signature of competition and hence are inappropriate to test the naturalization hypothesis. These authors advocate for quantifying relatedness based on the phylogenetic distance between exotic and local native species as a stronger framework to link invader characteristics and community properties. The key assumption that phylogenetic distance correlates with ecological distance is also contentious, largely because the strength of niche conservatism is known to vary across clades (Wienset al. 2010). Evaluating this assumption empirically requires not only testing whether phylogenetic relatedness describes the degree of niche overlap in resource use - a fundamental premise of the naturalization hypothesis - but also whether it describes the extent to which close relatives share the traits required to thrive in a particular environment, a major assumption of the preadaptation hypothesis.

The ecological context is also critical to reconciling the opposing predictions regarding the relationship between phylogenetic distance and invasiveness. Specifically, the naturalization hypothesis assumes that competition controls community composition. While competition can certainly prevent the success of some introduced species (Levineet al. 2004), whether this is the primary mechanism controlling biological invasions remains controversial. One reason is that many exotic species attain higher success in human-altered environments, where competitive regimes may have been changed by frequent disturbances and the extirpation of many native species (Elton 1958; Bartomeuset al. 2012; Cadotte et al. 2017; Sol et al.2017b). In these environments, the success of the species may largely depend on the ability of individuals to cope with new challenges to which they have had little opportunity to adapt, potentially favoring preadapted species (Sol \& Maspons 2016; Sol et al. 2017b). However, previous attempts to reconcile the naturalization and preadaptation hypotheses have focused on the influence of the spatial scale of analysis rather than on the environmental contexts where either competition or filtering should be more relevant. Park et al. (Parket al. 2020), for example, recently suggested that at small spatial scales, competitive exclusion inhibits alien invasions, but these effects are attenuated at larger spatial scales because of greater environmental heterogeneity. Thus, spatial scale provides an alternative solution to Darwin's naturalization conundrum.

Here, we provide an empirical assessment of Darwin's naturalization conundrum for birds. A recent analysis by Redding et al. (Reddinget al. 2019) revealed that the biotic environment had a relatively weak effect on establishment success of birds compared to other factors such as propagule pressure or the species' life history. After accounting for these other factors, however, the probability of establishment increased with the presence of closely related species in the region. While these findings are consistent with the preadaptation hypothesis, it remains to be shown whether and how the phylogenetic structure of the recipient communities influences the success of invaders once established. This is a crucial gap, because the impact of exotic species largely depends on the extent to which the species increases in numbers and expands across different habitats.

Using published data on well-surveyed avian communities along gradients of human-related disturbances (Sol et al. 2020b), we assess whether phylogenetic relatedness to native species predicts invasion success, and whether the predictive power varies with the scale of analysis and/or the degree human-related disturbances. Our analyses are based on $>1,300$ estimations of invasion success for 63 exotic species -including all the most invasive birds and invaded regions worldwide (Dataset 1 ). Because the same species was frequently 
introduced to different locations, and because most locations were subject to several introductions and contained communities with different species and exposed to different levels of disturbance (Fig. 1 ), our dataset provides a unique opportunity to clarify Darwin's naturalization conundrum. In addition, we make use of the increased availability of avian functional trait data sets describing fine-scale variation in trophic niche, resource acquisition behaviors and response to novel environments (Pigot et al. 2020; Solet al. 2020b), allowing us to go beyond a mere assessment of phylogenetic relatedness between invaders and their community neighbors to assess whether phylogenetic patterns reflect ecological differences.

\section{Methods}

Survey data. We used information on well-surveyed avian communities from published studies and our own field work (Sol et al. 2020b, c). The term community is used to describe assemblages within a region that could be unambiguously assigned to one of five land-use classes: native vegetation, rural, little urbanized (e.g. urban parks), moderately urbanized (suburbs) and highly urbanized (city centers). Our criteria to accept data were that, at each study region, bird species were exhaustively surveyed and that at least one of the communities was invaded by one or more non-indigenous bird species. The resulting database comprised information on 1367 species - 63 non-indigenous in one or more communities - in 277 communities from 50 study regions. In 47 of these study regions, surveys were conducted both in urbanized areas and in the surrounding non-urbanized areas, allowing us to assess the effect of human-related disturbances on invasiveness. The entire dataset is available in Sol et al. (2020a).

Introduced species information. We extracted information on bird species introduced and established in regions outside their native range from the Global Avian Invasion Atlas GAVIA (Dyer et al. 2017), the most complete dataset of historical introductions currently available. The dataset covers $>27,000$ introductions of 971 bird species and also provides information of the year of introduction for established exotics.

Invasion success. We used two classic measures of invasion success: 1) the presence/absence of the invader in communities from a same region, 2) the abundance of exotic species relative to native species within the invaded communities. The Gavia dataset (see above) confirmed that the exotic species analyzed were introduced sufficiently time ago (range from 1853 to 1975) to make their abundances little influenced by time lags or introduction effort. The density of occurrences of an animal in a given place may however be influenced by their size, either directly (e.g. by increasing the demands of food) or indirectly (by correlating with the fast-slow continuum of life history variation). We evaluated the importance of body size by including it as a co-variate in the models (see below).

Phylogenetic information. We extracted from the BirdTree database (http://www.birdtree.org) (Jetz et al. 2012), two samples of 5,000 phylogenies that included all native and exotics species included in this study. Each sample was based two alternative backbone topologies: Hackett et al. (2008) and Ericson et al. (2006). Phylogenetic distances were estimated based on two summary trees calculated from both samples. These were computed as the maximum clade credibility tree using the program TreeAnnotator (included in the package BEAST v1.8.0) (Drummond et al. 2012). Because results from both phylogenies were highly coincident, we only present the results of analyses based on Ericson et al. (2006).

Functional traits information. We described the foraging niche of species based on three types of functional traits extracted from Pigot et al. (Pigot et al. 2020): 1) eight morphological traits (all log-transformed), 2) seven diet categories, and 3) 30 categories of foraging behavior collected from the literature, as described in Sol et al. (Sol et al. 2020b). Diet and foraging behavior were described as fuzzy variables, ranking each category from 0 to 10 as a function of the degree of use (Pigot et al. 2020). In addition, we extracted information on relative brain size, resource niche breadth and brood value from Sol et al. (Sol et al. 2014a, 2020b) and Sayol et al. (Sayol et al. 2016). To estimate niche breadth, we used Rao's quadratic entropy (De Cáceres et al. 2011), based on the functional distances matrix (see below) derived from niche information.

Phylogenetic and functional distance metrics. We used two complementary metrics of phylogenetic distance, the average and nearest phylogenetic distance between each exotic species and all the native species of the community (Tucker et al. 2017). For each invader from a given site, we calculated the mean (MPD) and 
nearest phylogenetic distance (NPD) between invaders and all native species of each community regardless of whether the exotic species was present or not (see justification below). Summary trees were pruned down to the species present in the communities and phylogenetic distances among species were calculated by means of the function "cophenetic" in the R package "Picante" (Kembel et al. 2010). To consider the fact that most native species in the community were rare, we used weighted versions of MPD and NPD in which the distance of the invader to each native species was multiplied by $1-A b_{r}$, where $A b_{r}$ is the relative abundance of the invader in the community. This increases the distance of rarer species relative to more common species. Functional distances were estimated the same way, but applying Gower's distance (Gower 1971) to morphological, diet and foraging behavior traits (the last two coded as fuzzy variables).

Spatial scale and biases. We used our community dataset to assess how the nearest phylogenetic distance between exotic and native species varied with spatial scale (i.e. community, region, country and continent). We used simulations to explore whether spatial patterns were affected by the increase in species with scale or by the fact that exotic and native species came from different regions. The former was investigated by randomly sampling without replacement pairs of species in different numbers (from 1 to 125) from the entire avian phylogeny $(\mathrm{n}=9993)$. We compared all species as well as the subset that were introduced in regions outside their native range and the subset of them that have become successfully established in the new region. The analysis of geographic effects was conducted comparing species within and across biogeographic realms that have been either donors (Palearctic) or receptors of invaders (Nearctic and Australian).

\section{Modelling invasion success as a function of phylogenetic and functional distance.}

To test whether phylogenetic distance predicted invasion success, we asked how mean phylogenetic distance (MPD) and nearest taxon phylogenetic distance (NTD) affected our two measures of invasion success by means of generalized linear mixed models (BPMM, hereafter). We built the models in the R packages MCMCglmm (Hadfield 2010) and BRMS (Bürkner 2017), which implement Bayesian generalized linear mixed models by means of a Markov chain Monte Carlo process. To test whether phylogenetic distance influenced the likelihood that the exotic occurred in a community, the occurrence of exotic species was modelled by means of BPMMs with binomial distribution and logit link, including either MPD or NTD (or related abundance-weighted metrics and metrics based on functional distances) as fixed predictor and study site, species (as some species occurred in more than one community) and phylogeny as random factors. The inclusion of study site as a random effect was important because it allowed modelling the likelihood that the exotic was present in a community to be evaluated within each study site, ensuring thus that the absence of the invaders did not result from the fact that the species had never been introduced in the region. To test whether phylogenetic distance influenced the relative abundance of the invaders within a community, we restricted the analyses to communities with two or more exotic species and used BPMMs with Gaussian structure of errors. The fixed and random effects were the same used to model occurrence with the only difference that study site was replaced by community as random factor. In this way comparisons were made within communities instead of across communities. For each BPMM model we ran two independent runs with 200,000 generations, from which a $10 \%$ was discarded as "burn in". Convergence and good mixing of each of the MCMC chains was assessed visually, plotting the traces of each of the model parameters. We re-ran all models including the interaction between phylogenetic distance and habitat, and the potential confounds of body size and native species richness. Based on a model selection approach, habitat was simplified to two categories according to the degree of human-related alterations: habitats with high levels of alterations (highly and moderately urbanized habitats) and habitats with low levels alterations (little urbanized, rural and natural habitats).

Sampling procedure to study the effect of species richness on phylogenetic distances. We randomly selected pairs of species without replacement for different sample sizes (from 10 to 150 pairs) and extracted the nearest phylogenetic distance from the matrix of phylogenetic distances for the entire avian phylogeny. We repeated the sampling 1,000 times, and represented the results as means and standards errors of the mean. The sampling was conducted for all birds, as well for the subset of species that were introduced in regions outside their native range and those from the subset that became established at least once (data from GAVIA 
(Dyer et al. 2017)). The approach was also used to show that whether introduced and established species are a non-random subset of all avian species.

Ecological validation of phylogenetic distance. We evaluated the assumption that phylogenetic distance is a good surrogate of functional distance in two ways. First, we used the package "geomorph" (Adams \& OtárolaCastillo 2013)to estimate the overall phylogenetic signal by means of a multivariate version of Blomberg's $K$ (Adams 2014). Major axes of niche variation were described by means of a Principal Coordinates Analysis (PCoA), based on Gower's generalized distance. Second, we used Mantel correlations to compare phylogenetic distances with functional distances (Mouquet et al. 2012). This analysis was conducted both on the entire dataset and splitting it in the main clades where exotics were represented. Finally, we used a multi-mantel test to test whether, besides describing the resource niche, phylogenetic relatedness also contained information on the degree of similarity in response traits (i.e. relative brain size, resource niche breadth and brood value).

\section{Results and Discussion}

Our analyses do not support that the probability of observing the signature of environmental filtering and competition is scale-dependent (Park et al. 2020). We found that the phylogenetic distance of exotic species to the closest coexisting native relative was, on average, three times larger at the local community level than at the continental level (Fig. 2A ), consistent with previous studies (Park et al. 2020). Yet a similar pattern was found when we instead estimated the minimum phylogenetic distance between each exotic species and the native species from communities where those exotic species are absent (Fig. 2B ). Thus, it is unlikely that the pattern is a signature of environmental filtering. A more plausible alternative is that the increase in species richness at larger spatial scales increases the probability of including a closely related exotic and native species (Fig. 2C ).

Given the risk that results are biased by sampling artifacts, we opted by addressing the conundrum at lower spatial scales and ask whether the success of the invader in a community is influenced by the degree of phylogenetic relatedness with native neighbors. Under the naturalization hypothesis, for example, we should expect that within any region exotic species will be restricted to those communities where they do not find close relatives. Instead, the preadaptation hypothesis predicts a preference for communities with close relatives. As some exotic species were absent from certain communities despite being well-established in nearby communities (505 out of 1,359 cases), we tested whether the probability that an exotic species occurred in a community was related to the phylogenetic distance with the native species from the community. Despite previous studies suggesting that the naturalization hypothesis should be favored at smaller spatial scales, we found no evidence that the presence of close relatives competitively exclude exotic species. On the contrary, we found that exotic species were more likely to be present in communities containing native species that are on average more closely related (Fig. 3A, B ).

One possible explanation for the failure to support the naturalization hypothesis is that native species may reduce the success of exotic species but rarely prevent invasion (Levine et al. 2004). Thus, the relative abundance of an exotic species in the community may be a more informative measure of invasion success to address the naturalization hypothesis. Based on estimations of exotic species abundance in communities where they were established ( $\mathrm{N}=792)$, we again found no evidence that exotic species more distantly related to native residents reach higher relative abundances than exotic species with closer relatives (Fig 3C, D ). Rather, exotic species abundances marginally decreased with the mean and nearest phylogenetic distance to native species. Although most native species in the studied communities had low relative abundances (Sol et al. 2020b), and thus may be unlikely to inhibit invasions even if the exotic species have similar resource needs, the conclusion does not change when using mean and nearest phylogenetic distances weighted by their relative abundance (Fig $\mathbf{3 E}-\mathbf{H}$ ).

The lack of support for the naturalization hypothesis may reflect that in birds competition plays a minor role in influencing invasion success, relative to other intrinsic or environmental factors (Duncan et al. 2003). One reason is that exotic species tend to attain higher success in disturbed habitats, mostly urbanized habitats (Sol et al. 2020b), where competitive regimes have frequently been altered by the extinction of native 
species (Bartomeus et al. 2012). These human-altered habitats often impose strong environmental filtering, as only a few species have the adaptations needed to live in artificial environments frequently disturbed by human activities (Sol et al.2014b). Adding the interaction between phylogenetic distance and degree of habitat alteration improves the fit of previous models of invasion success (Table S1 ). When considering this interaction, we find support for the preadaptation hypothesis, with a negative relationship between invasion success and phylogenetic distance but only for human-altered environments, such as urbanized habitats (Fig. 4 ). Importantly, this pattern is not confounded by species richness (Tables S2-S3 ) because urbanized environments tend to contain fewer species than the surrounding non-urbanized environments (Solet al. 2020b).

While our results support the pre-adaptation hypothesis, they are not exempt of potential biases. An issue that may make the patterns difficult to interpret is that the subset of species that have been introduced and successfully established in regions outside their native range is a non-random sample of the entire avian phylogeny, a possibility well-documented in the literature (Duncan et al.2003). The clustering of introduced species within the avian phylogeny should increase the probability that a native species randomly selected from the avian phylogeny belongs to a different clade. This effect may be further accentuated by the fact that introduced species often come from distant regions. For example, if we compare the phylogenetic distances of pairs of species randomly sampled within and across the main bioregions involved as donors and recipients of invaders, we find that by chance the average distances across bioregions are higher than within regions for a same sample size (Fig 2D, E ). To better interpret the results, we assessed to what extent phylogenetic patterns reflect ecological differences.

The notion that phylogenetic patterns reflect ecological differences is supported by two lines of evidence. First, we found that the main axes of niche variation across all species-measured in terms of morphology, dietary use and foraging behavior (see methods) - showed clear evidence of phylogenetic effects (Fig. S1), supporting the existence of substantial niche conservatism. A Mantel test further confirmed that phylogenetic distance co-varied with niche differentiation $(P=0.001)$, although the analysis also showed that it only accounted for $31 \%$ of variation in the niche. Second, similar to phylogenetic distance, we found a negative relationship between niche distance and invasion success measured in terms of abundance (Fig. 4; Table S4 ). The relationship between invasion success and niche distance appears to primarily occur in human-altered environments (Fig. 4K,L,O,P ).

Instead, we found a different pattern when we examined the probability that the invader occurred in a community within a given region. In communities occupying less human-altered areas, this probability tended to increase with functional distance, suggesting that invaders prefer to settle in communities that lack species with similar niches (Fig. 4I-N, Table S5 ). The alternative that the pattern results from extinction of close-relatives by the invader is less likely because invaders rarely achieved higher densities in little disturbed environments. Moreover, native species are expected to be more competitive than invaders in these environments because they have had more opportunities to adapt (Sax \& Brown 2000; Sol et al.2012a).

The discrepancy between patterns may indicate that functional and phylogenetic distances contain different information. Phylogenetic distances among species reflect differences in the niche, but they also contain important information on traits related to the ability of the species to persist in novel environments (Fig. 5). While niche availability is arguably essential to proliferate in a new environment, response traits like behavioral plasticity and a future returns life history strategy can also be important in novel environments to buffer individuals against the demographic consequences of maladaptation (Solet al. 2012b). As response traits are particularly relevant to live in human-altered environments (Sol et al. 2014a), it might be that phylogenetic distance better captures differences among species that are relevant for the pre-adaptation hypothesis than for the naturalization hypothesis. This finding further highlights the importance of using functional traits to interpret phylogenetic patterns. It also suggests that the accuracy of predictive models should increase when including functional information, a possibility supported by our data (Table S1 ).

Our comprehensive analysis of phylogenetic relatedness between invaders and natives yielded no support for naturalization hypothesis. Previous works suggest that the failure to support the hypothesis reflects flaws in 
the analyses, like the adoption of inappropriate spatial scales or the use of taxonomic instead of phylogenetic information (Thuilleret al. 2010; Li et al. 2015). However, we analyzed well-surveyed communities within a phylogenetic framework, and considered possible sampling artifacts and a number of confounds such as the body size of the invader and the relative abundance of native close relatives. We also considered the possibility that native competitors do not exclude invaders but merely reduce their abundance. Despite all these improvements, we failed to find evidence that nonindigenous organisms are more successful in communities in which their close relatives are absent. While the role of competition in invasions remains debatable, our results fit better with growing evidence that, at least in birds (Duncan et al. 2003; Sol et al. 2012a), competition only plays a minor role in explaining invasion success. However, our finding that the probability that the invader occurred in a community increased with functional distance may suggest that invaders tend to avoid natural communities where they find species with similar niche requirements. While human-altered environments are novel for both invaders and native species, in natural environments the opportunity for adaptation is substantially higher for native species. These communities should thus exert stronger biotic resistance, which may in part explain why exotic species tend to avoid them (Lovell et al. 2021). However, our analyses indicate that the signatures of these effects are difficult to detect with phylogenetic relatedness.

Our analyses provide more credence for the pre-adaptation hypothesis, showing that phylogenetic distance is a good surrogate for both niche and functional response traits and that lower functional distances are also associated with enhanced invasion success. This results aligns with growing evidence showing that invasion success in birds critically depends on the possession of appropriate adaptations to flourish in human-altered communities, where biotic resistance has been reduced by the extinction of specialized competitors (Sol et al. 2012b). Because these adaptations are phylogenetically conserved (Sol et al. 2014a, 2017a, 2020b), it follows that the existence of phylogenetically close species in the communities can inform us on the likelihood that an invader establishes itself and spread in a new region. Our findings thus extend those of Redding et al. (Reddinget al. 2019) by showing that the presence of closely related species in a region not only increases the probability of establishment but also the abundance a species attains, a key metric of invasion success and a factor determining the impact of exotic species. This provides some of the clearest evidence to date that the presence of close relatives enhances - rather than inhibits - biological invasions. It remains to be shown whether this conclusion can be extrapolated to organisms with less ability than birds to sample habitats and select those that best match their phenotypes.

We therefore conclude that phylogenies can improve our ability to accurately predict the likelihood that a species proliferates when introduced to a new region. However, phylogenetic information is only useful to make predictions when considering the environmental context in which invasions take place, particularly the degree of human-related disturbances. Moreover, phylogenetic information should be combined with functional data to accurately predict invasiveness, as phylogenetic distances are not always an accurate surrogate of ecological distances. The rapid development of new statistical techniques to integrate phylogenetic and phenotypic information (Penone et al. 2014) provides new, largely unexplored opportunities to improve our ability to predict and prevent the success and impact of biological invasions.

\section{Acknowledgments}

We thank all authors who published the information on their intensive urban avian surveys. We are also indebted to Dario Moreira for helping assemble the dataset. We finally thank Phillip Clergeau, Mariana Villegas and Ivan Diaz for providing unpublished data, María Moirón, Gabriel Garcia-Peña, Miquel Vallllosera and Louis Lefebvre for insightful comments, Liam Revell for sharing scripts to plot phylogenies, and the R-team and package contributors for the $\mathrm{R}$ free software. We are also grateful to Natural History Museum Tring, American Museum of Natural History and numerous other research collections for access to specimens. Collection of functional trait data was supported by Natural Environment Research Council grants NE/I028068/1 and NE/P004512/1 (to JAT). This paper is part of the project CGL2013-47448-P from the Spanish Government (to DS). CG-L was supported by FONDECYT 11160271 and PIA/BASAL FB0002 from ANID, Chile. JGP was supported by a Juan de la Cierva Fellowship from the Ministry of Economy, 
Industry and Competitivity of Spain (FJCI-2014-20380).

Adams, D.C. (2014). A Generalized K Statistic for Estimating Phylogenetic Signal from Shape and Other High-Dimensional Multivariate Data. Syst Biol , 63, 685-697.

Adams, D.C. \& Otarola-Castillo, E. (2013). Geomorph: an r package for the collection and analysis of geometric morphometric shape data.Methods Ecol Evol , 4, 393-399.

Bartomeus, I., Sol, D., Pino, J., Vicente, P. \& Font, X. (2012). Deconstructing the native-exotic richness relationship in plants. Glob Ecol Biogeogr , 21, 524-533.

Blackburn, T.M., Lockwood, J.L. \& Cassey, P. (2009). Avian Invasions . Avian Invasions Ecol Evol Exot Birds . Oxford University Press.

Burkner, P.-C. (2017). Brms: An R Package for Bayesian Multilevel Models Using Stan. J Stat Softw , 80, $1-28$.

De Caceres, M., Sol, D., Lapiedra, O. \& Legendre, P. (2011). A framework for estimating niche metrics using the resemblance between qualitative resources. Oikos , 120, 1341-1350.

Cadotte, M.W., Campbell, S.E., Li, S.-P., Sodhi, D.S. \& Mandrak, N.E. (2018). Preadaptation and Naturalization of Nonnative Species: Darwin's Two Fundamental Insights into Species Invasion. Annu Rev Plant Biol , 69, 661-684.

Cadotte, M.W., Yasui, S.L.E., Livingstone, S. \& MacIvor, J.S. (2017). Are urban systems beneficial, detrimental, or indifferent for biological invasion? Biol Invasions , 19, 3489-3503.

Daehler, C.C. (2001). Darwin's Naturalization Hypothesis Revisited.Am Nat , 158, 324-330.

Darwin, C. (1859). On the Origin of Species by Means of Natural Selection. D Applet Co .

Diez, J.M., Sullivan, J.J., Hulme, P.E., Edwards, G. \& Duncan, R.P. (2008). Darwin's naturalization conundrum: Dissecting taxonomic patterns of species invasions. Ecol Lett , 11, 674-681.

Drummond, A.J., Suchard, M.A., Xie, D. \& Rambaut, A. (2012). Bayesian Phylogenetics with BEAUti and the BEAST 1.7. Mol Biol Evol , 29, 1969-1973.

Duncan, R.P., Blackburn, T.M. \& Sol, D. (2003). The Ecology of Bird Introductions. Annu Rev Ecol Evol Syst , 34, 71-98.

Dyer, E.E., Redding, D.W. \& Blackburn, T.M. (2017). The global avian invasions atlas, a database of alien bird distributions worldwide.Sci Data , 4, 170041.

Elton, C. (1958). The ecology of invasions by animals and plants . Chapman and Hall, London.

Ericson, P.G.P., Zuccon, D., Ohlson, J.I., Johansson, U.S., Alvarenga, H. \& Prum, R.O. (2006). Higherlevel phylogeny and morphological evolution of tyrant flycatchers, cotingas, manakins, and their allies (Aves: Tyrannida). Mol Phylogenet Evol , 40, 471-483.

Gower, J.C. (1971). A General Coefficient of Similarity and Some of Its Properties. Biometrics , 27, 857.

Hackett, S.J., Kimball, R.T., Reddy, S., Bowie, R.C.K., Braun, E.L., Braun, M.J., et al. (2008). A Phylogenomic Study of Birds Reveals Their Evolutionary History. Science (80- ), 320, 1763-1768.

Hadfield, J.D. (2010). MCMC Methods for Multi-Response Generalized Linear Mixed Models: The MCMCglmm R Package. J Stat Softw , 33, 1-22.

Hulme, P.E., Bacher, S., Kenis, M., Klotz, S., Kuhn, I., Minchin, D., et al. (2008). Grasping at the routes of biological invasions: A framework for integrating pathways into policy. J Appl Ecol , 45, 403-414.

Jetz, W., Thomas, G.H., Joy, J.B., Hartmann, K. \& Mooers, A.O. (2012). The global diversity of birds in space and time. Nature, 491, 444-448. 
Jiang, L., Tan, J. \& Pu, Z. (2010). An Experimental Test of Darwin's Naturalization Hypothesis. Am Nat , 175, 415-423.

Kembel, S.W., Cowan, P.D., Helmus, M.R., Cornwell, W.K., Morlon, H., Ackerly, D.D., et al. (2010). Picante: R tools for integrating phylogenies and ecology. Bioinformatics , 26, 1463-1464.

Lambdon, P.W. \& Hulme, P.E. (2006). How strongly do interactions with closely-related native species influence plant invasions? Darwin's naturalization hypothesis assessed on Mediterranean islands. J Biogeogr , 33, 1116-1125.

Levine, J.M., Adler, P.B. \& Yelenik, S.G. (2004). A meta-analysis of biotic resistance to exotic plant invasions. Ecol Lett , 7, 975-989.

Li, S. peng, Cadotte, M.W., Meiners, S.J., Hua, Z. shuang, Shu, H. yue, Li, J. tian, et al. (2015). The effects of phylogenetic relatedness on invasion success and impact: Deconstructing Darwin's naturalisation conundrum. Ecol Lett, 18, 1285-1292.

Lovell, R.S.L., Blackburn, T.M., Dyer, E.E. \& Pigot, A.L. (2021). Environmental resistance predicts the spread of alien species. Nat Ecol Evol .

Mouquet, N., Devictor, V., Meynard, C.N., Munoz, F., Bersier, L.-F.F., Chave, J., et al. (2012). Ecophylogenetics: Advances and perspectives. Biol Rev , 87, 769-785.

Park, D.S., Feng, X., Maitner, B.S., Ernst, K.C. \& Enquist, B.J. (2020). Darwin's naturalization conundrum can be explained by spatial scale. Proc Natl Acad Sci , 117, 10904-10910.

Penone, C., Davidson, A.D., Shoemaker, K.T., Di Marco, M., Rondinini, C., Brooks, T.M., et al. (2014). Imputation of missing data in life-history trait datasets: which approach performs the best? Methods Ecol Evol, 5, 961-970.

Pigot, A.L., Sheard, C., Miller, E.T., Bregman, T.P., Freeman, B.G., Roll, U., et al. (2020). Macroevolutionary convergence connects morphological form to ecological function in birds. Nat Ecol Evol , 4, 230-239.

Pysek, P., Jarosik, V., Hulme, P.E., Kuhn, I., Wild, J., Arianoutsou, M., et al. (2010). Disentangling the role of environmental and human pressures on biological invasions across Europe. Proc Natl Acad Sci , 107, $12157-12162$.

Pyšek, P. \& Richardson, D.M. (2010). Invasive Species, Environmental Change and Management, and Health. Annu Rev Environ Resour , 35, 25-55.

Redding, D.W., Pigot, A.L., Dyer, E.E., Şekercioğlu, Ç.H., Kark, S. \& Blackburn, T.M. (2019). Locationlevel processes drive the establishment of alien bird populations worldwide. Nature , 571, 103-106.

Romanuk, T.N., Zhou, Y., Brose, U., Berlow, E.L., Williams, R.J. \& Martinez, N.D. (2009). Predicting invasion success in complex ecological networks. Philos Trans $R$ Soc B Biol Sci, 364, 1743-1754.

Sax, D.F. \& Brown, J.H. (2000). The paradox of invasion. Glob Ecol Biogeogr , 9, 363-371.

Sayol, F., Maspons, J., Lapiedra, O., Iwaniuk, A.N., Székely, T. \& Sol, D. (2016). Environmental variation and the evolution of large brains in birds. Nat Commun , 7, 13971.

Shea, K. \& Chesson, P. (2002). Community ecology theory as a framework for biological invasions. Trends Ecol Evol , 17, 170-176.

Sol, D., Bartomeus, I., González-Lagos, C. \& Pavoine, S. (2017a). Urbanisation and the loss of phylogenetic diversity in birds. Ecol Lett , 20, 721-729.

Sol, D., Bartomeus, I. \& Griffin, A.S. (2012a). The paradox of invasion in birds: Competitive superiority or ecological opportunism? Oecologia , 169, 553-564. 
Sol, D., González-Lagos, C., Lapiedra, O. \& Díaz, M. (2017b). Why Are Exotic Birds So Successful in Urbanized Environments? In: Ecology and Conservation of Birds in Urban Environments (eds. Murgui, E. \& Hedblom, M.). Springer International Publishing, Cham, pp. 75-89.

Sol, D., Gonzalez-Lagos, C. \& Moreira, D. (2020a). Worldwide bird assemblages across urban-wildland gradients. Dryad, Dataset, https://doi.org/10.5061/dryad.2rbnzs'7jf .

Sol, D., González-Lagos, C., Moreira, D., Maspons, J. \& Lapiedra, O. (2014a). Urbanisation tolerance and the loss of avian diversity.Ecol Lett , 17, 942-950.

Sol, D., Lapiedra, O. \& Vilà, M. (2014b). Do close relatives make bad neighbors ? Proc Nat Acad Sci USA , 111, 534-535.

Sol, D. \& Maspons, J. (2016). Life History, Behaviour and Invasion Success. In: Biological Invasions and Animal Behaviour (eds. Weis, J.S. \& Sol, D.). Cambridge University Press, Cambridge, pp. 63-81.

Sol, D., Maspons, J., Vall-llosera, M., Bartomeus, I., Garcia-Pena, G.E., Pinol, J., et al. (2012b). Unraveling the life history of successful Invaders. Science (80- ), 337, 580-583.

Sol, D., Trisos, C., Murria, C., Jeliazkov, A., Gonzalez-Lagos, C., Pigot, A.L., et al. (2020b). The worldwide impact of urbanisation on avian functional diversity. Ecol Lett , 23, 962-972.

Sol, D., Trisos, C., Murria, C., Jeliazkov, A., Gonzalez-Lagos, C., Pigot, A.L., et al. (2020c). Worldwide bird assemblages across urban-wildland gradients . Ecol Lett. John Wiley \& Sons, Ltd.

Thuiller, W., Gallien, L., Boulangeat, I., de Bello, F., Munkemuller, T., Roquet, C., et al. (2010). Resolving Darwin's naturalization conundrum: A quest for evidence. Divers Distrib , 16, 461-475.

Tucker, C.M., Cadotte, M.W., Carvalho, S.B., Davies, T.J., Ferrier, S., Fritz, S.A., et al. (2017). A guide to phylogenetic metrics for conservation, community ecology and macroecology. Biol Rev , 92, 698-715.

Wiens, J.J., Ackerly, D.D., Allen, A.P., Anacker, B.L., Buckley, L.B., Cornell, H. V., et al. (2010). Niche conservatism as an emerging principle in ecology and conservation biology. Ecol Lett, 13, 1310-1324.

Figures

\section{Hosted file}

image1.emf available at https://authorea.com/users/377081/articles/510239-a-test-of-darwins-naturalization-conundrum-in-birds-reveals-enhanced-invasion-success-in-presence-ofclose-relatives

Figure 1. Variation in nearest phylogenetic distance (NPD) between exotic and native species across the avian phylogeny. Panels show (A), NPD of the exotic to native species from the recipient community (B), and the degree to which this NPD is larger or smaller than expected for the NPD between native species $(\mathrm{C})$. This latter difference is the fraction of NPDs estimated between all pairs of native species of the community that are smaller than the NPD observed for the exotic and native species of the communities. Thus, a large value means that exotic species are less closely related to native species than are native species with each other, while a low value means the contrary.

\section{Hosted file}

image2.emf available at https://authorea.com/users/377081/articles/510239-a-test-of-darwins-naturalization-conundrum-in-birds-reveals-enhanced-invasion-success-in-presence-ofclose-relatives

Figure 2. Scale-dependent sampling effects on phylogenetic distance. (A-B) Nearest phylogenetic distance (NPD) of introduced species (63 species successfully established in 50 regions Worldwide) to native species estimated at different spatial scales; (C) species randomly sampled from the entire avian phylogeny $(\mathrm{n}=9,993)$, the subset that has been introduced in regions outside their native range $(\mathrm{n}=962)$ and those 
from the subset that have become successfully established in the new region ( $\mathrm{n}=443)$; and (D-E) species of the biogeographic realm from where most introduced species originated (Palearctic) and realms where those have more frequently been introduced (Nearctic and Australian). Codes: IC = invaded communities, NIC $=$ non-invaded communities, $\mathrm{REG}=$ regional level, $\mathrm{COU}=$ country and $\mathrm{CON}=$ continental.

\section{Hosted file}

image3.emf available at https://authorea.com/users/377081/articles/510239-a-test-of-darwins-naturalization-conundrum-in-birds-reveals-enhanced-invasion-success-in-presence-of-

close-relatives

Figure 3. Relationship between invasion success and phylogenetic distance as a function of mean and nearest phylogenetic distance to native species while accounting for phylogenetic, species and community effects. Lines show the values predicted by Bayesian mixed models, and the lower and upper bounds represents the uncertainty interval of the prediction. Occurrence models implement Bernoulli structure of errors and are based on 1358 estimations of presence/absence of 69 invader species across 277 communities. Abundance models implement Gaussian error structures and are based on 792 estimations of 62 invader species in 213 communities where the invaders were present. All models are univariate, including as a predictor mean phylogenetic (MPD), nearest phylogenetic distance (NPD) or each of these predictors weighted by abundance of native species (denoted with the subscript $w$ ).

\section{Hosted file}

image4.emf available at https://authorea.com/users/377081/articles/510239-a-test-of-darwins-naturalization-conundrum-in-birds-reveals-enhanced-invasion-success-in-presence-ofclose-relatives

Fig. 4. Success of invaders as a function of mean and nearest phylogenetic and functional distance to native species. Red plots indicate habitats highly altered by human activities (i.e. highly and moderately urbanized habitats) and, blue plots indicate habitats with low levels of alteration (i.e. urban parks, rural areas and wildlands). The lines represent the values predicted by Bayesian mixed models, and the lower and upper bounds represents the uncertainty interval of the prediction (95\% credibility intervals). Details on the models are shown in Tables S2-S5.

\section{Hosted file}

image5.emf available at https://authorea.com/users/377081/articles/510239-a-test-of-darwins-naturalization-conundrum-in-birds-reveals-enhanced-invasion-success-in-presence-ofclose-relatives

Fig. 5. Relationship between pairwise phylogenetic and functional distances (foraging niche and response traits) between native species (a) and between native and exotic species (b). Dots are 10,000 randomly sampled pairwise comparisons. 